\title{
The role of arginine and arginine-metabolizing enzymes during Giardia - host cell interactions in vitro
}

\author{
Britta Stadelmann ${ }^{1}$, Kurt Hanevik ${ }^{2,3}$, Mattias K Andersson ${ }^{1}$, Oystein Bruserud ${ }^{2}$ and Staffan G Svärd ${ }^{1 *}$
}

\begin{abstract}
Background: Arginine is a conditionally essential amino acid important in growing individuals and under nonhomeostatic conditions/disease. Many pathogens interfere with arginine-utilization in host cells, especially nitric oxide (NO) production, by changing the expression of host enzymes involved in arginine metabolism. Here we used human intestinal epithelial cells (IEC) and three different isolates of the protozoan parasite Giardia intestinalis to investigate the role of arginine and arginine-metabolizing enzymes during intestinal protozoan infections.

Results: RNA expression analyses of major arginine-metabolizing enzymes revealed the arginine-utilizing pathways in human IECs (differentiated Caco-2 cells) grown in vitro. Most genes were constant or down-regulated (e.g. arginase 1 and 2) upon interaction with Giardia, whereas inducible NO synthase (iNOS) and ornithine decarboxylase (ODC) were up-regulated within $6 \mathrm{~h}$ of infection. Giardia was shown to suppress cytokine-induced iNOS expression, thus the parasite has both iNOS inducing and suppressive activities. Giardial arginine consumption suppresses NO production and the NO-degrading parasite protein flavohemoglobin is up-regulated in response to host NO. In addition, the secreted, arginine-consuming giardial enzyme arginine deiminase (GiADI) actively reduces T-cell proliferation in vitro. Interestingly, the effects on NO production and T cell proliferation could be reversed by addition of external arginine or citrulline.
\end{abstract}

Conclusions: Giardia affects the host's arginine metabolism on many different levels. Many of the effects can be reversed by addition of arginine or citrulline, which could be a beneficial supplement in oral rehydration therapy.

Keywords: Protozoa, Diarrhea, Citrulline, Caco-2, Nitric oxide

\section{Background}

Giardia intestinalis (a.k.a. G. lamblia and G. duodenalis), a protozoan parasite, causes diarrhea in a wide variety of host species [1]. Due to the broad spectrum of hosts and genetic differences the parasite is divided into 8 assemblages (A to $\mathrm{H}$ ) [2], of which two (A and B) are responsible for approximately 300 million cases of human giardiasis yearly [2]. Giardiasis was included into the WHO initiative for neglected diseases in 2004 [3]. Patients get infected upon ingestion of infectious cysts in contaminated food or water that release proliferating trophozoites in the duodenum, establishing intestinal infection [1]. Roughly half of the infections stay asymp-

\footnotetext{
* Correspondence: staffan.svard@icm.uu.se

${ }^{1}$ Department of Cell- and Molecular Biology, Uppsala University, BMC, Box 596, Uppsala SE-751 24, Sweden

Full list of author information is available at the end of the article
}

tomatic, whereas the other half results in disease. Symptoms of giardiasis include nausea, vomiting, epigastric pain and watery diarrhea [4], though duration and symptoms are highly variable. Giardiasis is associated with malabsorption, reduced growth and developmental retardation in children [5], irritable bowel syndrome, arthritis and chronic fatigue [6]. It is a multifactorial disease but most of the virulence factors remain unknown $[2,7]$.

G. intestinalis is able to degrade the amino acid arginine as an energy source via the arginine dihydrolase pathway [8] and two of the enzymes of this pathway, arginine deiminase (ADI) and ornithine carbamoyltransferase (OCT), are released upon Giardia-intestinal epithelial cell (IEC) interaction [9]. The parasite rapidly reduces the amount of arginine in the growth medium during in vitro growth [7], resulting in reduced proliferation of IECs. Infection of IECs with an assemblage A

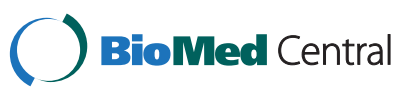


isolate of Giardia leads to a reduction of nitric oxide (NO) production by these cells [10] since arginine is also substrate for NO synthases (NOS). Giardia ADI was identified as the protein being responsible for a reduced NO response in in vitro interaction setups [9]. At least in vitro, $\mathrm{NO}$ acts cytostatic against G. intestinalis trophozoites and inhibits encystation and excystation [10], the two differentiation processes essential for infection. It plays a role in muscle relaxation and thus in mechanical parasite elimination by peristalsis $[11,12]$. Therefore reduction of the NO response of the host is in favor of Giardia growth. More recently, a NO-detoxifying enzyme (flavohemoglobin) was found in G. intestinalis, but its expression status upon host cell interaction has not been addressed yet $[13,14]$. Therefore it needs to be investigated how exactly Giardia interferes with the NO response of human IECs.

In mammalian cells, NO is formed either by NOS (eNOS, NOS3 in endothelial cells, nNOS, NOS1 in neuronal cells and iNOS, NOS2 in epithelial, endothelial and inflammatory cells) through conversion of arginine into citrulline and $\mathrm{NO}$ in an oxygen-dependent reaction, or through reduction of nitrite in various oxygenindependent ways [15]. NO has multiple roles in the human body, broadly taken together, as a cellular messenger and as an antimicrobial agent $[15,16]$. NO reacts with reactive oxygen intermediates, forming antimicrobial substances such as nitrogen dioxide, peroxynitrite, $\mathrm{S}$-nitrosothiols, dinitrogen trioxide and dinitrogen tetroxide that will cause damage in the cell wall, the DNA and the proteins of pathogens and also human cells [16]. However, effects of NO on Giardia trophozoites do not seem to be exerted by peroxynitrite [17].

Many pathogens are known to interfere with the host's arginine metabolism. Salmonella typhimurium, Mycobacterium tuberculosis, Helicobacter pylori, Trypanosoma brucei and T. cruzi, Toxoplasma gondii and Schistosoma mansoni are known examples of pathogens that compete with host NOS for their common substrate arginine via up-regulation of host arginases $[18,19]$. Some microorganisms are even known to consume arginine via their own arginases $[18,19]$. Thereby pathogens can reduce host $\mathrm{NO}$ production and increase polyamine synthesis, which is in favor of pathogen growth and survival. However, within such studies it has neither been addressed what functions argininemetabolizing enzymes apart from arginase or arginine transporters could play, nor has the direct consumption of arginine, or active detoxification of NO, by a pathogen been taken into account.

As shown in previous microarray studies [20] a variety of chemokines are induced upon Giardia-host cell interaction that would be potent in attracting immune cells such as $B$ and $T$ cells, dendritic cells, macrophages, monocytes, mast cells and neutrophils to the intestinal mucosa. For yet unknown reasons, Giardia-infection, however, leads only to a small increase in intra-epithelial lymphocytes and little or no mucosal inflammation in human giardiasis patients [2,21]. It has recently been shown that consumption of arginine and production of ammonia via Giardia ADI affects the phenotype and cytokine production of dendritic cells [22], but it is not known if arginine depletion affects other immune cells.

In the present study we show effects of the intestinal parasite Giardia on the innate and adaptive host immune response by focusing on the parasite's arginineconsuming ability and the enzyme ADI in particular. Effects on host cell's NO production, expression of arginine-consuming enzymes and $\mathrm{T}$ cell proliferation are shown. We also investigated a NO-detoxification system that the parasite induces NO-dependently upon host cell interaction.

\section{Results}

Expression of arginine-consuming enzymes in human IECs upon Giardia infection

Our earlier data showed that arginine is depleted in the growth medium already after $1-2 \mathrm{~h}$ of in vitro interaction between Giardia trophozoites and human IECs [7]. A number of enzymes and transporters are directly and indirectly involved in the arginine-metabolism of human cells (Figure 1). Pathogenic microbes are known to affect the expression of these enzymes, especially arginase 1 and 2 [18]. However, arginine-metabolism in human IECs is poorly characterized and it is not known how it is affected by Giardia infection. In order to study this, the expression of arginine-consuming enzymes was assessed in differentiated TC7 Caco- 2 cells, that exhibit small intestinal epithelial characteristics, by qPCR at time points $0,1.5,3,6$ and 24 h post in vitro Giardia infection. To study if different Giardia assemblages have different effects on the arginine metabolism we used trophozoites from three different isolates: WB (assemblage A), GS (assemblage B) and P15 (assemblage E) [2]. The assessed genes were the chemokine $c c l 20$ as positive infection control [20] and several arginine-consuming enzymes (see Figure 1 and 2, Additional file 1: Table S1). Except for cat2 and nos1, all tested genes were expressed in IECs, however, $a d c$, argI and nos3 only at very low levels (Additional file 1: Tables S2-S4). Most of the genes showed only slight changes in expression on RNA level over the $24 \mathrm{~h}$ experiment (Figure 2). The strong induction of $c c l 20$ already after $1.5 \mathrm{~h}$ of infection with Giardia trophozoites is in line with our earlier results [20]. None of the tested arginine-consuming enzymes were upregulated more than 2 times after $1.5 \mathrm{~h}$ of WB interaction. After 3 and $6 \mathrm{~h}, o d c$ and nos 2 were up-regulated more than 2 times in the WB interaction, but expression 


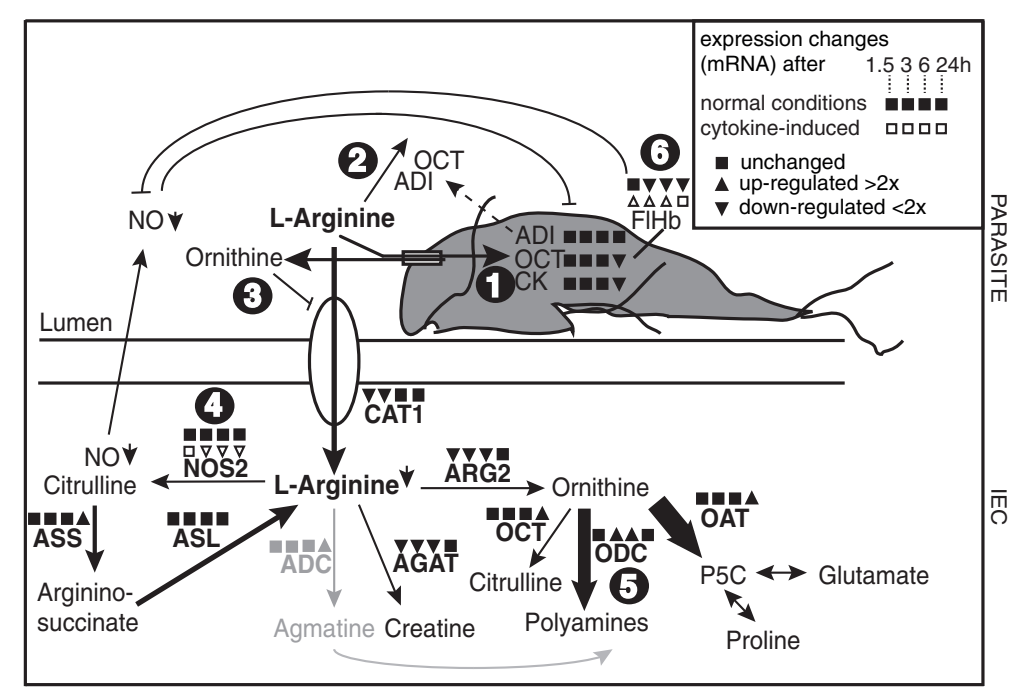

Figure 1 RNA expression changes of arginine-consuming enzymes upon Giardia-host cell interaction. Based on an interpretation of results from this and previous studies, the encircled numbers point out various ways by which Giardia interferes with the host immune response: (1) consumption of arginine via arginine-ornithine antiporter, (2) release of arginine-consuming ADI and OCT, (3) blocking of arginine-uptake into host cells by ornithine, (4) down-regulation of host iNOS, (5) up-regulation of host ODC, (6) up-regulation of parasite FlHb upon NO-stress. Human intestinal epithelial cells (Caco-2) were in vitro interacted with Giardia trophozoites and the expression changes of arginine-consuming enzymes were assessed by qPCR. Various enzymes involved in the arginine-metabolism of host cells and of Giardia are shown (adapted from Stadelmann et al 2012 [7]). Changes in expression after 1.5, 3, 6 and $24 \mathrm{~h}$ as compared to $0 \mathrm{~h}$ are indicated for interactions with the parasite isolate WB according to Figures 2 and 4 (square for no change, triangle pointing up for up-regulation, triangle pointing down for downregulation; cut-off value 2). Expression of inos and flhb in host cells that were stimulated with cytokines (TNF-a (200 ng/mL), IL-1a (200 ng/mL), IFN- $\gamma(500 \mathrm{ng} / \mathrm{mL}$ ) to produce nitric oxide is also shown (non-filled triangles for up- and down-regulation, non-filled square for no change). ADC, arginine decarboxylase; ADI, arginine deiminase; AGAT, arginine-glycine amidinotransferase; ARG, arginase; ASL, argininosuccinate lyase; ASS, argininosuccinate synthetase; CAT, cationic amino acid transporter; CK, carbamate kinase; FlHb, flavohemoglobin; NO, nitric oxide; NOS, nitric oxide synthase; OAT, ornithine aminotransferase; OCT, ornithine carbamoyl transferase; ODC, ornithine decarboxylase; p6C, $\Delta 1$-pyrroline-5-carboxylate.

dropped at $24 \mathrm{~h}$. The same observations were made in interactions with parasites of the isolates GS and P15. However, the effects on induction of $c c l 20$, nos 2 and odc were much more pronounced upon infection with the isolate GS than with WB and P15 (Figure 2). $\operatorname{arg1}$, arg2 and agat were down-regulated at all time points with a 4- (arg1), 3- (arg2) and 6.5-fold (agat) reduction at $6 \mathrm{~h}$ of WB interaction, followed by recovery to near basal expression levels after $24 \mathrm{~h}$. This profile was also seen in interactions with the two other isolates. Several other genes $(a d c, o a t, o c t)$ showed the same expression profiles with an initial decrease followed by an increase at $24 \mathrm{~h}$. Thus, upon depletion of arginine by Giardia trophozoites (after 1-2 h), expression levels of most host argininemetabolizing enzymes are reduced, independent of the parasite isolate. The results are summarized in Figure 1, which shows the complex gene expression changes occurring when Giardia trophozoites interact with host IECs.

\section{Effects of $G$. intestinalis on nitric oxide production of human IECs}

Inducible nitric oxide, iNOS, encoded by nos2, is a key enzyme in NO production during infections $[10,18]$. To further investigate the observed effects on the nos 2 expression and iNOS activity in host cells upon Giardia infection, effects of different arginine levels were assessed. The growth of IECs in low-arginine medium compared to growth with extra arginine $(0.4 \mathrm{mM}$ arginine added to the low-arginine medium) surprisingly showed that nos 2 was highly induced on the RNA level under low-arginine conditions (Figure 3a). The profile of nos2 induction in low-arginine medium was similar to the profile induced by Giardia infection with a peak of expression after $6 \mathrm{~h}$ (Figure 2). Strikingly, the level of expression upon parasite-interaction was lower than in the low-arginine medium. We therefore tested the hypothesis that Giardia can induce expression of nos 2 via arginine depletion, but at the same time also downregulate its expression. To test this hypothesis an alternative model was used, where nos 2 expression was first induced in HCT-8 cells by addition of cytokines (TNF- $\alpha$ (200 ng/mL), IL-1 $\alpha$ (200 ng/mL, IFN- $\gamma(500 \mathrm{ng} / \mathrm{mL})$ prior to Giardia infection ( $40 \mathrm{~h}$ later). Parasite addition clearly and strongly down-regulated the expression of nos2 (Figure 3b). Thus, Giardia can both induce and down-regulate expression of iNOS.

To assess the production of NO upon iNOS induction in Giardia-interacted human cells, the NO levels upon 

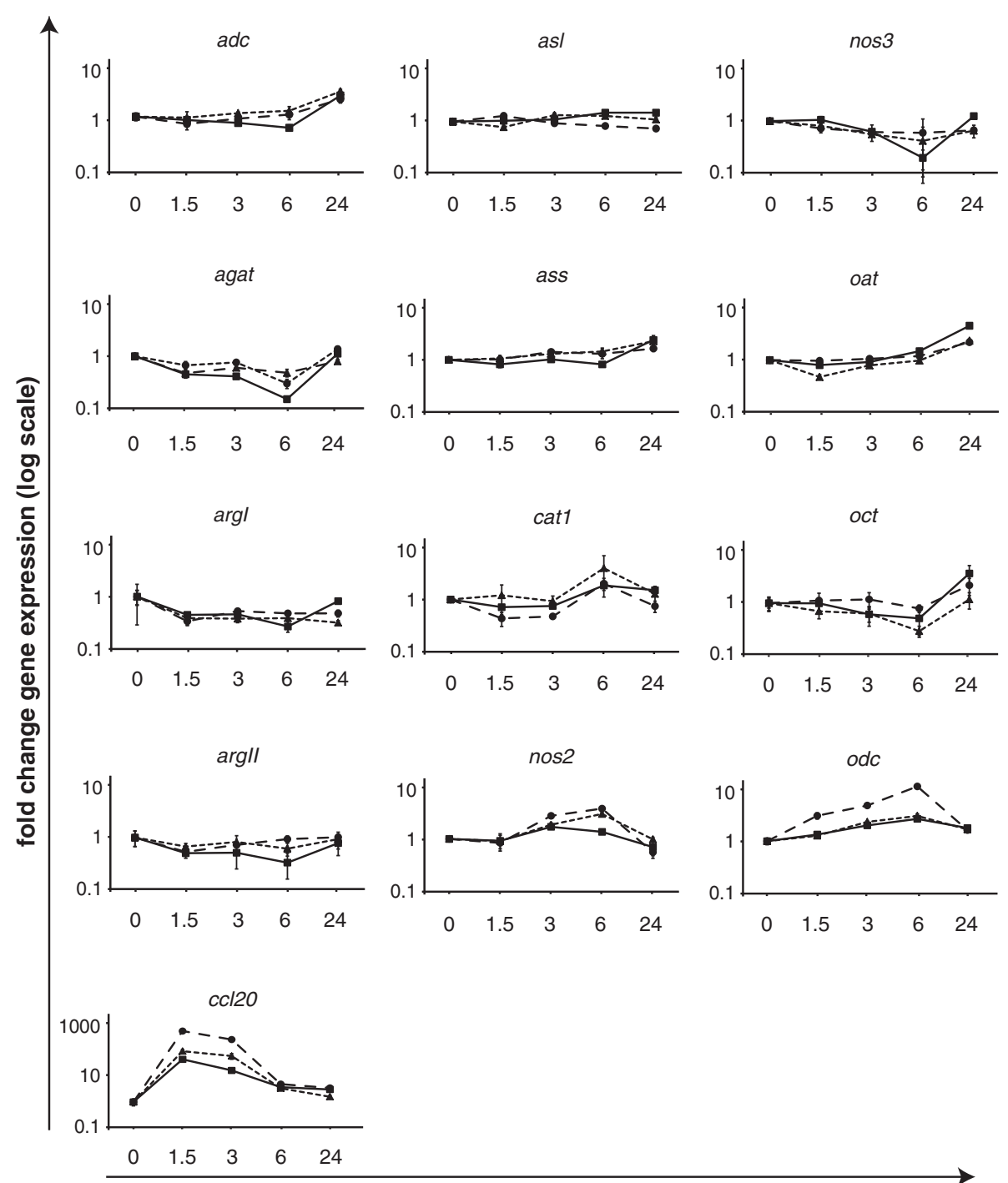

WB trophozoites

interaction time $(h)$

Figure 2 Expression of arginine-metabolizing enzymes in IECs upon Giardia infection. Differentiated Caco-2 IECs were in vitro infected with Giardia trophozoites of three different assemblages (isolates WB (squares), GS (circles) and P15 (triangles)) and expression of arginine-consuming enzymes in host cells was assessed after 0, 1.5, 3, 6 and $24 \mathrm{~h}$ on the RNA level by qPCR in technical quadruplicates. The data is presented as fold change gene expression in arbitrary units on a logarithmic scale as compared to $0 \mathrm{~h}$ expression. GAPDH was used as reference gene. In total 12 different arginine-consuming genes and the control gene $c c 120$ were assessed for their expression. Note the changed scale for $c c 120$. adc, arginine decarboxylase; agat, arginine-glycine amidinotransferase; arg, arginase; asl, argininosuccinate lyase; ass, argininosuccinate synthetase; cat, cationic amino acid transporter; ccl20, chemokine (C-C motif) ligand 20; nos, nitric oxide synthase; oat, ornithine aminotransferase; oct, ornithine carbamoyl transferase; odc, ornithine decarboxylase.

infection with isolates of three different assemblages of Giardia was assessed. Trophozoites of the isolates WB, GS and P15 were all able to completely suppress NO production of IECs and the IECs did not recover from this within 4 days, even though parasite survival is limited to roughly $24 \mathrm{~h}$ within the present interaction system (Figure 3c). Arginine added to physiological concentrations of $0.4 \mathrm{mM}$ could partially restore the $\mathrm{NO}$ production of parasite-interacted IECs (Figure 3d). Interestingly, the addition of citrulline, a metabolite of arginine, to a final concentration of $0.4 \mathrm{mM}$ could also restore the capability of IECs to produce NO upon Giardia infection (Figure 3d). Thus, Giardia can interfere with the innate host immune response by consuming arginine, the substrate of iNOS. Host cells try to compensate this by inducing iNOS, but the parasite can 
A

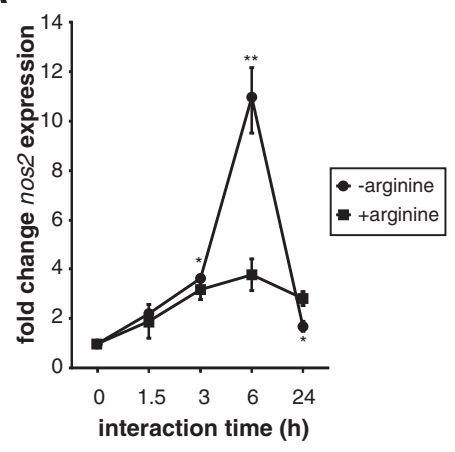

C

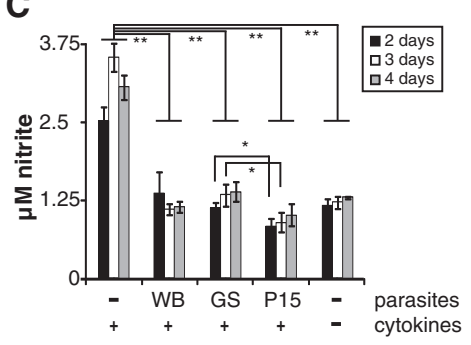

B

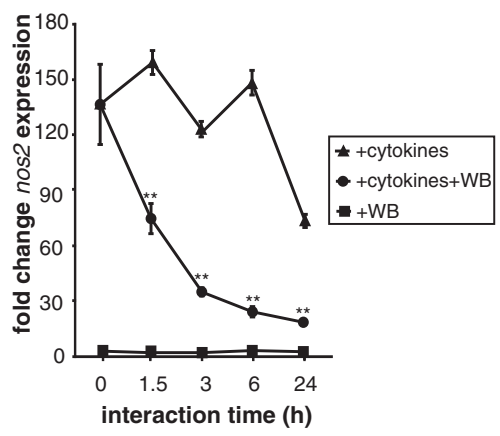

D

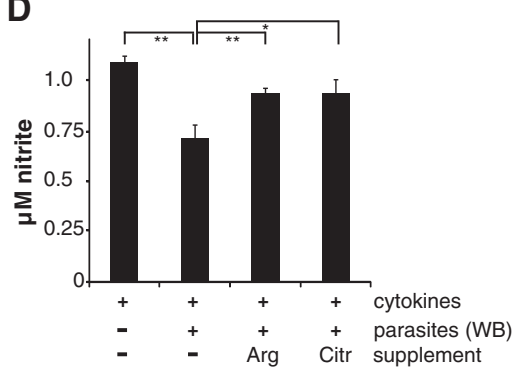

Figure 3 Giardia reduces host cell nitric oxide (NO) production. A, Expression changes of inducible nitric oxide synthase (nos2) in differentiated Caco-2 cells in medium with (+ arginine) and without (- arginine) arginine as assessed by qPCR in technical quadruplicates. Data is expressed as fold change expression compared to the $0 \mathrm{~h}$ timepoint. Significant expression changes compared to $0 \mathrm{~h}$ are indicated by asterisks. B, Expression changes of nos2 upon host cell (HCT-8) stimulation with cytokines (TNF-a (200 ng/mL), IL-1a (200 ng/mL), IFN- $(500 \mathrm{ng} / \mathrm{mL})$ ) and Giardia infection $40 \mathrm{~h}$ later. Data is expressed as fold change expression compared to the $0 \mathrm{~h}$ unstimulated control (squares). C, NO production of host cells (HCT-8) stimulated with cytokines $5 \mathrm{~h}$ after infection with Giardia trophozoites of 3 different isolates (WB, GS, P15). This experiment was repeated two times independently and lead to similar results. D, Giardia (isolate WB) infected host cells (HCT-8) were stimulated by cytokines to produce NO after $5 \mathrm{~h}$ of infection. Subsequently arginine (Arg) or citrulline (Citr) were added to $0.4 \mathrm{mM}$ after $1 \mathrm{~h}$ of interaction. NO production was measured $40 \mathrm{~h}$ later. The described experiment was repeated two times independently and lead to similar results. Significant differences in the figure are indicated by asterisks ( ${ }^{*}$ for $p<0.5$ and ${ }^{* *}$ for $p<0.01$ ).

also reduce the expression of iNOS, thereby affecting the host's NO production.

\section{Expression of enzymes in Giardia upon IEC infection}

Apart from expression changes in host IECs, we also assessed the response of Giardia enzymes that are directly or indirectly involved in arginine-metabolism upon host-interaction. The three main enzymes of arginine metabolism, ADI, OCT and CK, had previously been shown to be initially up-regulated but later downregulated after host cell infection [23]. To further investigate this and include also later time points of interaction, trophozoites of the isolate $\mathrm{WB}$ were let to interact with differentiated Caco-2 cells for 1.5, 3, 6 and $24 \mathrm{~h}$. Corresponding parasite controls were conducted in host cell medium. Thereby, the parasite genes adi, oct and $c k$ were down-regulated on the RNA level compared to control samples already after 1.5-3 h (Figure 4, Additional file 1: Table S5). Thus, the down-regulation of the expression of parasite arginine metabolizing enzymes occurs at the same time as arginine is depleted in the growth medium, showing that not only host cells, but also parasite cells, are changing the expression of arginine-consuming enzymes upon interaction.

\section{Further NO-defending mechanisms of Giardia}

To test whether the parasite G. intestinalis also uses other mechanisms than consuming arginine and changing iNOS expression to combat the antimicrobial host-NO response, the expression of the NO-detoxifying enzyme flavohemoglobin $[13,14](\mathrm{FlHb})$ was assessed.

Giardia trophozoites were interacted with host IECs that were previously induced to produce NO by addition of cytokines (as described above). Compared to non-stimulated IEC controls, Giardia trophozoites upregulated $\mathrm{FlHb}$ expression on the RNA and protein level (Figure 5) when the IECs produced NO. This could provide another layer of $\mathrm{NO}$ protection for the parasite (Figure 1).

\section{Proliferation of arginine-deprived PBMC}

To assess effects of the local arginine-deprivation caused by Giardia on infiltrating lymphocytes, peripheral blood mononuclear cells (PBMCs) were incubated 


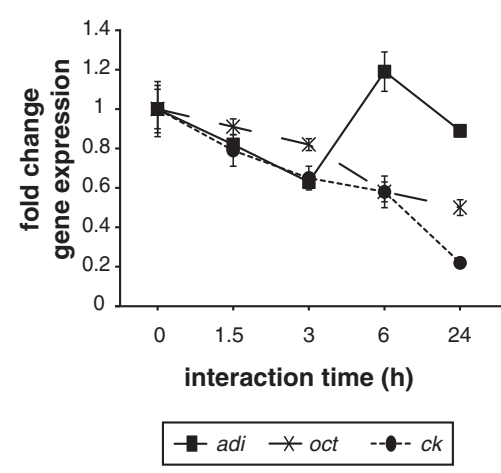

Figure 4 Expression of arginine-metabolizing enzymes in Giardia trophozoites upon host-cell interaction. Differentiated Caco-2 IECS were infected with Giardia trophozoites (isolate WB) and expression of arginine-consuming enzymes (adi, arginine deiminase; oct, ornithine carbamoyltransferase; ck, carbamate kinase) was assessed at 0, 1.5, 3, 6 and $24 \mathrm{~h}$ on the RNA level by qPCR in technical quadruplicates. GL50803_17364 was used as reference gene. Fold change gene expression is shown with substracted medium effects.

in a concentration series of GiADI and stimulated by $\mathrm{T}$ cell activating anti-CD3 and anti-CD28 antibodies. The GiADI used for this experiment was produced in and purified from Giardia trophozoites and exhibited in vitro arginine-degrading activity as earlier described [7]. There was a dose-dependent repression of T-cell specific PBMC proliferation upon addition of GiADI to PBMCs that reached full effect at $5 \mu \mathrm{g} / \mathrm{mL}$ GiADI (data not shown). This GiADI-dependent repression of PBMC proliferation after T-cell specific stimulation could be reduced by the addition of arginine to $0.4 \mathrm{mM}$, and partially also by citrulline to $0.4 \mathrm{mM}$ (Figure 6). Respective buffer and denatured protein controls showed no significant inhibitory effects (Figure 6).

\section{Discussion}

The fact that Giardia consumes arginine as an energy source is well-known $[8,24]$. However, possible roles of arginine in the pathophysiology of the host have only recently caught attention [2,7]. Within the present study we therefore assessed the effects of Giardia-infection of human IECs on the expression of arginine-metabolizing enzymes. Since gene expression changes during the very first hours of infection can only be studied in vitro, we used the in vitro interaction system described in [2,7]. We focused on changes on the RNA level since we earlier identified large changes in host cell gene expression already after $1.5 \mathrm{~h}$ [20] and early changes of gene expression are best detectable on the RNA level. As shown in Figure 2, most of the host arginine-metabolizing genes were unaffected or slightly down-regulated upon Giardia-infection. nos2, the inducible form of the nitric oxide synthases (iNOS), was induced after 3 and $6 \mathrm{~h}$ of parasite interaction, but down-regulated after $24 \mathrm{~h}$ to
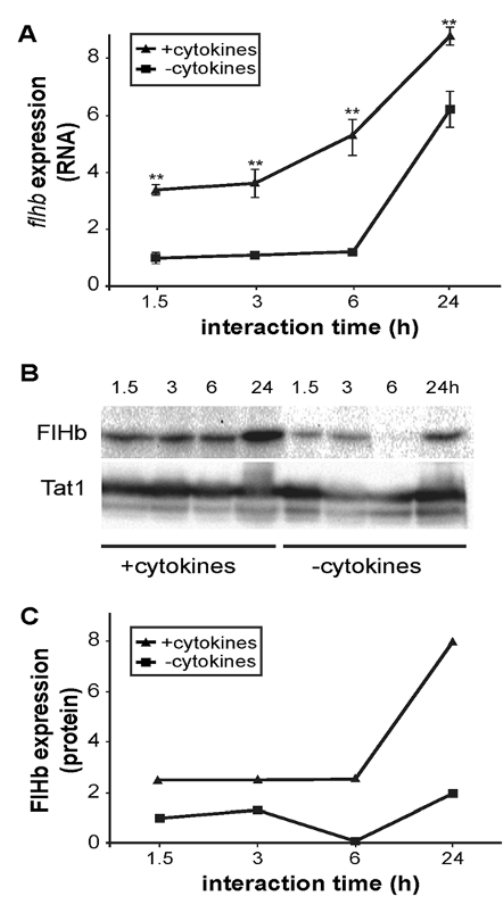

Figure 5 Giardia up-regulates flavohemoglobin upon nitric oxide (NO) stress. Human intestinal epithelial cells (HCT-8) were stimulated for $\mathrm{NO}$ production by addition of cytokines (TNF-a (200 ng/mL), IL-1a (200 ng/mL), IFN- $\gamma(500 \mathrm{ng} / \mathrm{mL}))$. Giardia trophozoites of the isolate WB were added to the NO-producing host cells and to control cells after $40 \mathrm{~h}$. Samples were measured for expression of the NO-detoxifying protein flavohemoglobin $(\mathrm{FlHb})$ at indicated time points. A, Upon interaction with NO-producing cells FlHb was induced in trophozoites on the RNA level compared to the control gene GL50803_17364 as assessed by GPCR in technical quadruplicates. This highly significant difference is indicated by asterisks. B, Western blot detecting the expression of $\mathrm{FlHb}$ and the control protein Tat1 in Giardia upon interaction with HCT-8 cells with and without NO-induction. C, Quantification of the Western blot bands (B) by image J software clearly shows the induction of $\mathrm{FlHb}$ protein in Giardia trophozoites upon interaction with NO-induced host cells. The results are representative for similar results obtained by three independent experiments.

levels slightly lower than before interaction. We detected a similar induction of nos 2 expression in IECs cultivated without arginine as compared to cells grown with arginine, peaking at $6 \mathrm{~h}$ (Figure 3). When we induced iNOS expression in host IECs by addition of cytokines, Giardia trophozoites immediately down-regulated this expression (Figure 3), which is not in accordance with earlier results [10], however, fewer parasites per IEC, a different cell line (HT-29), different cytokine concentrations and another experimental approach with measurements after $18 \mathrm{~h}$ was used in that study. Thus, Giardia infection on one hand immediately induces iNOS by arginine-depletion, but at the same time there are also iNOS down-regulating mechanisms in the parasite. Accordingly, iNOS expression was down-regulated in 


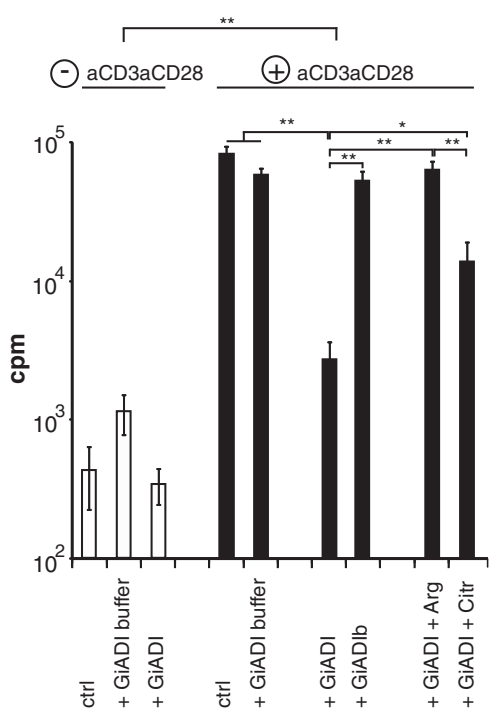

Figure 6 Giardia ADI reduces PBMC proliferation through arginine consumption. The secreted Giardia protein ADI (GiADI) was expressed and purified from Giardia WB trophozoites. T-cells of human PBMCs of 5 blood donors were stimulated to proliferate by activating anti-CD3 and anti-CD28 (indicated by + ) and the proliferation was assessed upon incorporation of ${ }^{3} \mathrm{H}$-labelled thymidine, as depicted in counts per minute (cpm) in logarithmic scale. T-cell stimulation was successful and not inhibited by buffer controls (+ GiADI buffer) or addition of heat denatured GiADI (+ GiADlb). GiADI $(5 \mu \mathrm{g} / \mathrm{mL})$ clearly reduced PBMC proliferation after T-cell specific stimulation, an effect that could be reversed by addition of arginine (+ GiADI + Arg) and partially also by its metabolite citrulline (+ GiADI + Citr). Significant differences are indicated by $*(p<0.5)$ and ${ }^{* *}(p<0.01)$.

Giardia-infected calves in vivo on RNA and protein level after several weeks of infection $[25,26]$.

As shown in Figure 2, the host's cationic amino acid transporter 1 (CAT1), used for arginine-uptake into host cells, was down-regulated in an early response (1.5-3 h), but up-regulated after $6 \mathrm{~h}$ of interaction. This response of co-induction of nos 2 and cat1, combined with a downregulation of arginases, ensures that the host cells take up sufficient arginine for NO synthesis (Figure 1). Such a coinduction has been shown in murine macrophages [27] though it was claimed that CAT2 and not CAT1 is upregulated together with iNOS [28]. Within our study we could not detect expression of cat 2 in IECs.

A variety of microbes are known to affect the host's immune response by down-regulating host NO production, either via an up-regulation of host arginases or expression of their own arginases $[18,19]$ that compete for consumption of arginine with iNOS. As shown in Figure 2, host arginases were not up-regulated upon IEC-Giardia interaction in vitro. However, later time points than $24 \mathrm{~h}$ were not included due to limitations of the setup. Whether arginase expression is up-regulated at later time points in vivo is, to the best of our knowledge, unknown. Interestingly however, the expression of ODC, a downstream enzyme of arginase, was highly up-regulated at all times (Figure 2). This might lead to a shift of the arginine-flux away from iNOS into polyamine synthesis [7].

Giardia infection leads to an increased expression of $o d c$, inos and cat1 during the first hours of interaction, whereas other arginine-consuming enzymes are downregulated or constant. We therefore studied how the parasite can defend itself against this initial response. As shown in Figure 3, we were able to see a NO reduction similar to Giardia-infection of IECs [10] and addition of Giardia ADI expressed in E. coli [9]. Moreover, this effect was observed for parasites of 3 different isolates (from humans (WB and GS) and pigs (P15)). Interestingly, the observed effect could be reverted by addition of arginine and also by its metabolite citrulline. This finding is interesting with regards to use of citrulline as a supplement in rehydration therapy, as discussed below.

In addition to actively taking up arginine, Giardia consumes arginine also indirectly via the secretion of the enzymes ADI and OCT that degrade arginine to ornithine via citrulline [9]. Ornithine, secreted as a final product of arginine fermentation via an arginineornithine antiporter [29], has been shown to block arginine transport into IECs [30] (Figure 1). Upon interaction with host cells, the expression of arginine-consuming enzymes ADI, OCT and CK was down-regulated already after $1.5 \mathrm{~h}$ on the RNA level (Figure 4), which is in accordance to Ringqvist et al [23]. As suggested, the expression of these enzymes might be increased shortly after secretion (15 minutes after host-parasite interaction), but is down-regulated at later time points due to depletion of arginine in the medium and due to a possible switch to glucose as main energy source [7].

It is not known to date, whether Giardia leads to a systemic arginine-deficiency in patients, this needs to be followed up. However, the local reduction of arginine levels by $G$. intestinalis could have additional consequences on the host response, the immune response in particular, since replication and infiltration of immune cells in the intestine might be blocked. It was recently shown that the phenotype and cytokine production of dendritic cells can be affected by the argininemetabolizing enzyme ADI from Giardia [22]. Even though a variety of cytokines are induced upon Giardiahost cell interaction, there is no strong intestinal inflammatory response exerted. Nevertheless, a role of $\mathrm{T}$ cells in elimination of Giardia infection has been shown by Singer and Nash in mice [31]. A specific T cell proliferative response to Giardia proteins in humans has been reported [32] and it has been suggested that ADI can inhibit this response [33]. Indeed, we could show that the secreted Giardia protein ADI is capable of reducing the 
human PBMC proliferative response after $\mathrm{T}$ cell specific stimulation (Figure 6) and thereby probably inhibit a strong immune response in vivo. Maximum effects were gained with a concentration of $5 \mu \mathrm{g} / \mathrm{mL}$ GiADI or above. This amount of GiADI is reasonable for mimicking the in vivo situation, since Giardia produces and releases ADI constantly. This finding is also in accordance with the decreased proliferation shown for $\mathrm{T}$ cells cultured without L-arginine [34] that was shown to be due to down-regulation of the CD3zeta chain of the T cell receptor. Furthermore, we were able to completely revert the observed reduction in $\mathrm{T}$ cell specific stimulated PBMC proliferation by addition of arginine to physiological levels (Figure 6).

Arginine is part of certain oral rehydration formulations used for treating diarrhea. However, adverse reactions such as osmotic diarrhea and excessive liver urea production $[35,36]$ are not in favor of such a therapy. In addition, arginine supplementation therapy might also be beneficial for the growth of Giardia itself, since the parasite uses arginine as an energy source. For these reasons we also tested the arginine-metabolite citrulline as an alternative supplementary therapy within this study. Citrulline can be reverted into arginine by argininosuccinate synthetase (ASS) and argininosuccinate lyase (ASL), which were both expressed in the IECs used for this study, but not in Giardia. It is not clear up to now if citrulline can also be reconverted into arginine in vivo by human cells such as IECs, dendritic cells and $\mathrm{T}$ cells. However, in children up to 3 years the argininereconverting enzymes ASS and ASL are actively expressed in IECs [37]. In addition, ASS and ASL were detected in the canine intestine [38] and it was shown that citrulline supplementation leads to increased arginine levels also in IECs in adult mice [39]. Thus it is likely, that citrulline conversion into arginine is possible in the intestine of human adults. In accordance to this, we could show that citrulline is capable of reversing all the described arginine-dependent effects on NOproduction and $\mathrm{T}$ cell proliferation that Giardia is exerting (Figures $3 \mathrm{~d}$ and Figure 6). Interestingly, the arginine-dependent block of proliferation that was shown to be induced in IECs upon Giardia infection, could also be reverted by citrulline [7]. Therefore citrulline, an abundant component of watermelon, should gain more attention in the future to be used as a supplement in oral rehydration solutions. It could help generating a proper immune response against Giardia and inhibiting pathophysiological effects in the intestinal epithelium that are caused by arginine-consumption of Giardia.

\section{Conclusion}

The findings presented here, and earlier data, clearly show that Giardia interferes with a proper host immune response of the host intestinal epithelium on the innate and adaptive immunity level by affecting arginine in the intesine on multiple levels (Figure 1). The parasite consumes arginine as an energy source $[7,24]$ and thereby the substrate for NOS [10]. Giardia trophozoites release arginine-consuming enzymes ADI and OCT [9] and ornithine that blocks the host cell transporter for arginine uptake [29]. Expression of iNOS is initially induced but reduced by the parasite at later stages of infection. Expression of ODC is also induced, further shifting arginine-flux away from iNOS. Flavohemoglobin expression is induced in Giardia early upon NO-stress [13]. Dendritic cell cytokine production [22] and T cell proliferation is affected due to reduced arginine-availability. All the observed effects might not be overwhelmingly strong by themselves, but the sum of them will certainly protect the parasite from the host's response.

\section{Methods}

\section{Ethics statement}

Individuals contributing peripheral blood mononuclear cells (PBMC) for the study of $\mathrm{T}$ cell proliferation gave written consent in a standard form upon registration as blood donors. The study and consent procedure was approved by the Regional Committee for Ethics in Medical Research (REK), Bergen, Norway.

\section{Reagents and cell culture}

If not stated otherwise, all chemicals and reagents were purchased from Sigma Chemical Co, USA. G. intestinalis trophozoites (strain WB, clone C6 (ATCC30957), strain GS, clone H7 (ATCC50581) and strain P15 were maintained in Giardia growth medium, TYDK, as described in Stadelmann et al [7]. G. intestinalis trophozoites were used for interaction with human intestinal epithelial cells (IECs) when reaching confluence. They were washed in PBS twice and counted before dilution in complete DMEM (high-glucose DMEM with $10 \%$ fetal bovine serum (Gibco ${ }^{\circ}$ Invitrogen, Paisley, UK), $4 \mathrm{mM} \mathrm{L-}$ glutamine, $1 \times$ MEM non-essential amino acids, $160 \mu \mathrm{g} / \mathrm{mL}$ streptomycin and $160 \mathrm{U} / \mathrm{mL}$ penicillin G) and addition to IECs at indicated numbers. IEC cell lines $\mathrm{CaCo}-2$, clone TC7 and HCT-8 (ATCC CCL-244), were maintained as described in Stadelmann et al [2,7], at $37^{\circ} \mathrm{C}, 5 \%$ $\mathrm{CO}_{2}$, in humid atmosphere, the same conditions that were applied for interaction experiments.

\section{Giardia - IEC interaction: gene expression}

For assessment of gene expression of G. intestinalis infected human IECs, Caco-2 cells were cultured in T25 tissue culture flasks 21 days post confluence with medium changes twice per week to allow differentiation [9]. Before addition of parasites, IECs were washed in prewarmed DMEM. Subsequently, $7.1 \times 10^{6}$ parasites 
were added to culture flasks. Control bottles contained complete DMEM with parasites only. Samples for RNA extraction were taken after $0,1.5,3,6$ and $24 \mathrm{~h}$ of interaction. Therefore, parasites were detached on ice for 10 min, supernatant was removed and human IECs were washed twice in cold PBS before being taken up in $1 \mathrm{~mL}$ TRIZOL $^{\circ}$ (Invitrogen) and stored at $-20^{\circ} \mathrm{C}$ until further RNA extraction. To extract parasite RNA and protein, the supernatant of interactions including detached parasites was centrifuged at $500 \times \mathrm{g}, 4^{\circ} \mathrm{C}$, for $10 \mathrm{~min}$ and taken up in $1 \mathrm{~mL}$ TRIZOL ${ }^{\circ}$.

To assess the expression status of arginine-consuming enzymes in human IECs as well as parasite genes induced upon interaction, RNA was extracted from each respective interaction sample according to the standard TRIZOL protocol. cDNA was prepared and $\mathrm{qPCR}$ performed as described in Stadelmann et al [7]. Primers are given in Additional file 1: Table S1. Human gapdh (X01677) and G. intestinalis WB ribosomal protein S26 (GL50803_17364) were used as reference genes $[7,23]$. Host cell gene expression was related to the $0 \mathrm{~h}$ expression value. Parasite gene expression was expressed relative to the expression of parasites kept in complete DMEM.

\section{Gene expression in low-arginine medium}

To assess the expression of nos 2 under low arginineconditions, Caco-2 cells were differentiated as described above in complete DMEM over $21 \mathrm{~d}$ in culture flasks, with medium changes twice per week. Thereafter, cells were washed in PBS and the medium was changed to low-arginine medium (RPMI 1640 (with L-glutamine, without arginine, leucine, lysine or phenol red) supplemented with $10 \%$ fetal bovine serum, $160 \mu \mathrm{g} / \mathrm{mL}$ streptomycin, $160 \mathrm{U} / \mathrm{mL}$ penicillin $\mathrm{G}, 0.4 \mathrm{mM}$ L-lysine and $0.38 \mathrm{mM}$ L-leucine) or low-arginine medium supplemented with $0.4 \mathrm{mM}$ L-arginine as described in Stadelmann et al, 2012 [7]. Samples for RNA extraction were taken after $0,1.5,3,6$ and $24 \mathrm{~h}$ and nos 2 expression assessed by qPCR as described above.

\section{Giardia - IEC interaction: nitric oxide production}

To compare the amounts of nitric oxide $(\mathrm{NO})$ production upon interaction of IECs with different parasite isolates, $5 \times 10^{6}$ HCT- 8 cells were seeded in T25 tissue culture flasks and grown to pre-confluence for 5 days. $3 \times 10^{6}$ parasites (isolates WB, GS and P15) were added to each flask, including PBS controls. $5 \mathrm{~h}$ later cells were stimulated for $\mathrm{NO}$ production by adding cytokines (TNF- $\alpha$ (200 ng/mL), IL-1 $\alpha$ (200 ng/mL; Santa Cruz Biotechnology), IFN- $\gamma(500 \mathrm{ng} / \mathrm{mL}$; Santa Cruz Biotechnology)). Supernatants for NO measurement were taken after 2, 3 and 4 days of incubation, centrifuged for $10 \mathrm{~min}$ at $500 \times \mathrm{g}$ and supernatants stored at $-20^{\circ} \mathrm{C}$ until measurement. Therefore triplicates of each sample were measured. $100 \mu \mathrm{L}$ of the supernatant was reduced by $100 \mu \mathrm{L}$ of nitrate reductase mix including $0.06 \mathrm{U} / \mathrm{mL}$ nitrate reductase from Arabidopsis thaliana, $2.5 \mu \mathrm{M}$ FAD and $100 \mu \mathrm{M}$ NADPH in $\mathrm{K}_{2} \mathrm{HPO}_{4}(50 \mathrm{mM}$, pH 7.5) in 96 well plates, for $3 \mathrm{~h}$ at $37^{\circ} \mathrm{C}$. Subsequently, nitrite was detected by the Griess Reagent System (Promega, Madison, WI) according to manufacturer's instructions. The absorbance at $540 \mathrm{~nm}$ was read in a Multiskan MS Plate Reader and nitrite concentrations were calculated according to a standard curve. To revert the parasite induced effects on NO production, arginine or citrulline were added to $0.4 \mathrm{mM}$ final concentration in the same setup after $1 \mathrm{~h}$ of interaction between HCT cells and WB parasites. Supernatants for NO measurement were taken after $40 \mathrm{~h}$ of incubation and prepared and measured accordingly.

\section{Giardia-IEC interaction upon iNOS induction: gene expression}

In order to assess gene and protein expression changes in parasite trophozoites upon host-cell induced NOstress, HCT-8 cells were seeded in T25 culture flasks and cultivated and stimulated for NO-production with cytokines as described above. After $40 \mathrm{~h}$, parasites were added to $7 \times 10^{6}$ parasites per bottle. Host cells and interacted parasites were harvested after 0, 1.5, 3, 6 and $24 \mathrm{~h}$. As controls, samples were also taken from host cells that were stimulated with cytokines but not interacted with parasites, or not stimulated with cytokines but interacted with parasites for the same time intervals. To assess the expression of inos in CaCo-2 cells, these were taken up in $1 \mathrm{~mL}$ TRIZOL $^{\circ}$ for further RNA extraction and qPCR as described above. Parasites were taken up in $1 \mathrm{~mL}$ TRI$\mathrm{ZOL}^{\circ}$ for subsequent RNA and protein extraction. cDNA synthesis and qPCR were performed as described above.

To assess expression status of Giardia flavohemoglobin also on protein level, Western blot was performed. Protein from interaction setups was extracted from TRIZOL samples and Western blot performed by blocking of protein-containing BioTraceTM PVDF membrane (Pall Corporation, Pensacola, FL) in 3\% non-fat milk in PBST. Proteins were detected by use of rabbit anti-Giardiaflavohemoglobin (by courtesy of Alessandro Giuffrè, University of Rome, Italy) 1:5'000 diluted in 0.3\% non-fat milk in PBST including also a loading control (mouse monoclonal Tat1, 1:5,000 [40]). Secondary HRP-labeled antibodies anti-rabbit and anti-mouse were diluted 1:8,000 and 1:10,000 respectively in $0.3 \%$ non-fat milk in PBST. HRP was detected using Western Lightning ECL Pro (PerkinElmer Inc, Waltham, MA USA) and chemoluminescence detected in a Universal Hood III (Bio Rad). Semi-quantitative comparison of bands was performed by ImageJ 1.32j. 


\section{PBMC acquisition and culture}

Peripheral blood mononuclear cells (PBMCs) were isolated by density gradient separation using Lymphoprep (Axis-Shield, Oslo, Norway) from buffycoats obtained from 5 healthy blood donors after routine blood donation. PBMC were washed in $\mathrm{NaCl}$ before cells were dissolved in X-vivo 15 serum-free culture medium supplemented with L-glutamine, gentamicin and phenol red (BioWhittaker, Walkersville, MA, USA). The freshly isolated PBMC $\left(8 \times 10^{4}\right.$ cells/well; $200 \mu \mathrm{l}$ medium/well $)$ were cultured in 96-well U-bottom microtiter plates in X-vivo 15 medium. Cells were cultured in medium alone, or in the presence of intact functional GiADI (produced, purified and tested as described in Jerlstrom-Hultqvist et al [41]), heat denatured $\left(80^{\circ} \mathrm{C}\right.$ for $\left.10 \mathrm{~min}\right)$ GiADI (GiADIb), as well as an equal dilution of BSA $1 \mu \mathrm{g} / \mathrm{mL}$ and PreScission enzyme containing buffer used for elution of GiADI, in combinations with $0.4 \mathrm{mM}$ arginine or citrulline and T-cell stimulatory anti-CD3 (mouse IgE moab; CLB-T3/4.E; final concentration $0.3 \mu \mathrm{g} / \mathrm{mL}$ ) and anti-CD28 (mouse IgG1moab; CLB-CD28/1; final concentration $0.8 \mu \mathrm{g} / \mathrm{mL}$ ) from the Central Laboratory of the Netherlands Red Cross Blood Transfusion Services (Amsterdam, The Netherlands). Cultures were performed in triplicates for 6 days at $37^{\circ} \mathrm{C}$ in a humidified atmosphere of $5 \% \mathrm{CO}_{2}$.

\section{PBMC proliferation assay}

Cellular proliferative responses were measured by the incorporation of ${ }^{3} \mathrm{H}$-thymidine into newly synthesized DNA by conventional proliferation assay [42]. After 5 days of culture cells were pulsed with $37 \mathrm{kBq} /$ well of ${ }^{3} \mathrm{H}$-thymidine (Perkin Elmer, Boston, MA, USA) and harvested $18 \mathrm{~h}$ later onto glass-fibre pads. Amounts of DNA-incorporated radioactivity were determined by liquid scintillation counting. Proliferation was determined as counts per minute (cpm).

\section{Data analysis}

If not mentioned otherwise, all data were analyzed using Microsoft Office Excel 2010. Figures were prepared in Adobe Illustrator CS4. Statistical analyses were performed by two-tailed student's $\mathrm{t}$-test ( $\mathrm{p}$-value $<0.5$, significant; $<0.01$, highly significant).

\section{Additional file}

Additional file 1: Describes primers used in RT-PCR analyses (Table S1), expressions of arginine consuming enzymes in IECs interacting with strain WB (Table S2), GS (Table S3) and P15 (Table S4). Table S5 describes expression of arginine-consuming enzymes in Giardia WB trophozoites during interaction with IECS.

\section{Competing interests}

The authors declare that they have no competing interests.

\section{Authors' contributions}

BS planned and performed all experiments, except the T cell proliferation study, and wrote the manuscript. $\mathrm{KH}$ and OB performed the T cell study. MA performed the NO reduction experiment. SGS conceived the study, participated in its design and wrote the final version of the manuscript. All authors read and approved the final manuscript.

\section{Acknowledgements}

Steinar Sørnes is thanked for assistance in the lab. Alessandro Giuffre, University of Rome, is acknowledged for sharing of the anti-flavohemoglobin antibody. This study was supported by VR-M and FORMAS (Sweden).

\section{Author details}

${ }^{1}$ Department of Cell- and Molecular Biology, Uppsala University, BMC, Box 596, Uppsala SE-751 24, Sweden. Institute of Medicine, University of Bergen, Bergen N-5021, Norway. ${ }^{3}$ Centre for Tropical Infectious Diseases, Department of Medicine, Haukeland University Hospital, Bergen N-5021, Norway.

Received: 28 June 2013 Accepted: 6 November 2013

Published: 14 November 2013

\section{References}

1. Svard SG, Hagblom P, Palm JE: Giardia lamblia - a model organism for eukaryotic cell differentiation. FEMS Microbiol Lett 2003, 218:3-7.

2. Ankarklev J, Jerlstrom-Hultqvist J, Ringqvist E, Troell K, Svard SG: Behind the smile: cell biology and disease mechanisms of Giardia species. Nat Rev Microbiol 2010, 8:413-422.

3. Savioli L, Smith H, Thompson A: Giardia and cryptosporidium join the 'neglected diseases initiative. Trends Parasitol 2006, 22:203-208.

4. Adam R: Biology of Giardia lamblia. Clin Microbiol Rev 2001, 14:447-475.

5. Ali S, Hill D: Giardia intestinalis. Curr Opin Infect Dis 2003, 16:453-460.

6. Wensaas KA, Langeland N, Hanevik K, Morch K, Eide GE, Rortveit G: Irritable bowel syndrome and chronic fatigue 3 years after acute giardiasis: historic cohort study. Gut 2012, 61:214-219.

7. Stadelmann B, Merino MC, Persson L, Svard SG: Arginine consumption by the intestinal parasite giardia intestinalis reduces proliferation of intestinal epithelial cells. PLoS One 2012, 7:e45325.

8. Schofield PJ, Costello M, Edwards MR, O'Sullivan WJ: The arginine dihydrolase pathway is present in Giardia intestinalis. Int J Parasitol 1990, 20:697-699.

9. Ringqvist E, Palm JE, Skarin H, Hehl AB, Weiland M, Davids BJ, Reiner DS, Griffiths WJ, Eckmann L, Gillin FD, Svard SG: Release of metabolic enzymes by Giardia in response to interaction with intestinal epithelial cells. Mol Biochem Parasitol 2008, 159:85-91.

10. Eckmann L, Laurent F, Langford T, Hetsko M, Smith J, Kagnoff M, Gillin F: Nitric oxide production by human intestinal epithelial cells and competition for arginine as potential determinants of host defense against the lumen-dwelling pathogen Giardia lamblia. J Immunol 2000, 164:1478-1487.

11. Li E, Zhou P, Singer SM: Neuronal nitric oxide synthase is necessary for elimination of Giardia lamblia infections in mice. J Immunol 2006 176:516-521.

12. Li E, Zhao A, Shea-Donohue T, Singer SM: Mast cell-mediated changes in smooth muscle contractility during mouse giardiasis. Infect Immun 2007 75:4514-4518

13. Mastronicola D, Testa F, Forte E, Bordi E, Pucillo LP, Sarti P, Giuffre A: Flavohemoglobin and nitric oxide detoxification in the human protozoan parasite Giardia intestinalis. Biochem Biophys Res Commun 2010, 399:654-658.

14. Rafferty S, Luu B, March RE, Yee J: Giardia lamblia encodes a functional flavohemoglobin. Biochem Biophys Res Commun 2010, 399:347-351.

15. Lundberg JO, Weitzberg E, Gladwin MT: The nitrate-nitrite-nitric oxide pathway in physiology and therapeutics. Nat Rev Drug Discov 2008, 7:156-167.

16. Jones ML, Ganopolsky JG, Labbé A, Wahl C, Prakash S: Antimicrobial properties of nitric oxide and its application in antimicrobial formulations and medical devices. App/ Microbiol Biotechnol 2010, 88:401-407.

17. Fernandes PD, Assreuy J: Role of nitric oxide and superoxide in Giardia lamblia killing. Braz J Med Bio/ Res 1997, 30:93-99.

18. Das P, Lahiri A, Chakravortty D: Modulation of the arginase pathway in the context of microbial pathogenesis: a metabolic enzyme moonlighting as an immune modulator. PLoS Pathog 2010, 6:e1000899. 
19. Morris SM Jr: Arginine: master and commander in innate immune responses. Sci Signal 2010, 3:pe27.

20. Roxström-Lindquist K, Ringqvist E, Palm D, Svärd S: Giardia lamblia-induced changes in gene expression in differentiated Caco-2 human intestinal epithelial cells. Infect Immun 2005, 73:8204-8208.

21. Cotton JA, Beatty JK, Buret AG: Host parasite interactions and pathophysiology in Giardia infections. Int J Parasitol 2011, 41:925-933.

22. Banik S, Renner Viveros P, Seeber F, Klotz C, Ignatius R, Aebischer T: Giardia duodenalis arginine deiminase modulates the phenotype and cytokine secretion of human dendritic cells by depletion of arginine and formation of ammonia. Infect Immun 2013, 81:2309-2317.

23. Ringqvist $E$, Avesson $L$, Soderbom F, Svard SG: Transcriptional changes in Giardia during host-parasite interactions. Int J Parasitol 2011, 41:277-285.

24. Schofield PJ, Kranz P, Edwards MR: Does Giardia intestinalis need glucose as an energy source? Int J Parasitol 1990, 20:701-703.

25. Dreesen L, Rinaldi M, Chiers K, Li R, Geurden T, Van den Broeck W, Goddeeris B, Vercruysse J, Claerebout E, Geldhof P: Microarray analysis of the intestinal host response in Giardia duodenalis assemblage $E$ infected calves. PLoS One 2012, 7:e40985.

26. Mokrzycka M, Kolasa A, Kosierkiewicz A, Wiszniewska B: Inducible nitric oxide synthase in duodenum of children with Giardia lamblia infection. Folia Histochem Cytobiol 2010, 48:191-196.

27. Nicholson B, Manner CK, Kleeman J, MacLeod CL: Sustained nitric oxide production in macrophages requires the arginine transporter CAT2. J Biol Chem 2001, 276:15881-15885

28. Yeramian A, Martin L, Serrat N, Arpa L, Soler C, Bertran J, McLeod C, Palacin M, Modolell M, Lloberas J, Celada A: Arginine transport via cationic amino acid transporter 2 plays a critical regulatory role in classical or alternative activation of macrophages. J Immunol 2006, 176:5918-5924.

29. Knodler LA, Schofield PJ, Edwards MR: L-arginine transport and metabolism in Giardia intestinalis support its position as a transition between the prokaryotic and eukaryotic kingdoms. Microbiology 1995, 141(Pt 9):2063-2070

30. Cendan JC, Souba WW, Copeland EM, Lind DS: Characterization and growth factor stimulation of L-arginine transport in a human colon cancer cell line. Ann Surg Oncol 1995, 2:257-265.

31. Singer SM, Nash TE: T-cell-dependent control of acute Giardia lamblia infections in mice. Infect Immun 2000, 68:170-175.

32. Hanevik K, Kristoffersen EK, Sornes S, Morch K, Naess H, Rivenes AC, Bodtker JE, Hausken T, Langeland N: Immunophenotyping in post-giardiasis functional gastrointestinal disease and chronic fatigue syndrome. BMC Infect Dis 2012, 12:258

33. Ropolo AS, Touz MC: A lesson in survival, by Giardia lamblia. ScientificWorldJournal 2010, 10:2019-2031.

34. Zea AH, Rodriguez PC, Culotta KS, Hernandez CP, DeSalvo J, Ochoa JB, Park HJ, Zabaleta J, Ochoa AC: L-Arginine modulates CD3zeta expression and T cell function in activated human T lymphocytes. Cell Immunol 2004, 232:21-31.

35. Grimble GK: Adverse gastrointestinal effects of arginine and related amino acids. J Nutr 2007, 137:1693S-1701S.

36. Bahri S, Zerrouk N, Aussel C, Moinard C, Crenn P, Curis E, Chaumeil JC, Cynober L, Sfar S: Citrulline: from metabolism to therapeutic use. Nutrition 2013, 29:479-484.

37. Kohler ES, Sankaranarayanan S, van Ginneken CJ, van Dijk P, Vermeulen JL, Ruijter JM, Lamers WH, Bruder E: The human neonatal small intestine has the potential for arginine synthesis; developmental changes in the expression of arginine-synthesizing and -catabolizing enzymes. BMC Dev Biol 2008, 8:107.

38. Daniel EE, Wang YF, Salapatek AM, Mao YK, Mori M: Arginosuccinate synthetase, arginosuccinate lyase and NOS in canine gastrointestinal tract: immunocytochemical studies. Neurogastroenterol Motil 2000, 12:317-334.

39. Wijnands KA, Vink H, Briede JJ, van Faassen EE, Lamers WH, Buurman WA, Poeze M: Citrulline a more suitable substrate than arginine to restore NO production and the microcirculation during endotoxemia. PLoS One 2012, 7:e37439.

40. Woods A, Sherwin T, Sasse R, MacRae TH, Baines AJ, Gull K: Definition of individual components within the cytoskeleton of Trypanosoma brucei by a library of monoclonal antibodies. J Cell Sci 1989, 93(Pt 3):491-500.

41. Jerlström-Hultqvist J, Stadelmann B, Birkestedt S, Hellman U, Svärd S: Plasmid vectors for proteomic analyses in Giardia: purification of virulence factors and analysis of the proteasome. Eukaryot Cell 2012, 11:864-873.

42. Wendelbo $\mathrm{O}$, Bruserud $\mathrm{O}$ : Functional evaluation of proliferative $\mathrm{T}$ cell responses in patients with severe $T$ lymphopenia: characterization of optimal culture conditions and standardized activation signals for a simple whole blood assay. J Hematother Stem Cell Res 2003, 12:525-535.

doi:10.1186/1471-2180-13-256

Cite this article as: Stadelmann et al:: The role of arginine and argininemetabolizing enzymes during Giardia - host cell interactions in vitro. BMC Microbiology 2013 13:256.

\section{Submit your next manuscript to BioMed Central and take full advantage of:}

- Convenient online submission

- Thorough peer review

- No space constraints or color figure charges

- Immediate publication on acceptance

- Inclusion in PubMed, CAS, Scopus and Google Scholar

- Research which is freely available for redistribution 